\title{
Personal susceptibility to pitch
}

\author{
G. A. HODGSON and H. J. WHITELEY \\ Cardiff Royal Infirmary and Welsh National School of Medicine, Cardiff
}

Hodgson, G. A., and Whiteley, H. J. (1970). Brit. J. industr. Med., 27, 160-166. Personal susceptibility to pitch. During the years 1957 to 1963,59 cases of hyperplastic squamous lesions were referred to us from a local patent fuel works where ovoids were made by fusing coal dust and pitch together by steam heat. We carried out a survey of all the personnel exposed to the pitch hazard and at the same time examined a control group of men of the same age group. The pitch workers examined totalled 144 and they were re-examined after a period of two years. The control group consisted of 263 persons selected from dermatological out-patients. The clinical findings were classified into six main groups: (1) benign proliferative lesions; (2) pre-malignant and malignant epidermoid lesions; (3) pitch acanthoma (pitch warts); (4) photo-sensitivity; (5) acneiform lesions; and (6) scrotal changes.

1. Benign proliferative lesions: There was little difference between the incidence of these lesions in the pitch workers and in the control group.

2. Pre-malignant and malignant epidermoid lesions: (a) Squamous keratoses were slightly more common in the pitch workers $(12 \%)$ than in the controls $(10 \%)$. (b) Chronic tar dermatosis was found in 5\% of pitch workers who had an exposure to pitch ranging from 30 to 50 years. No controls showed these changes. (c) Squamous-cell carcinoma incidence was $2.8 \%$ in pitch workers compared with $0.4 \%$ in the controls.

3. Pitch acanthoma: At the initial examination the incidence of pitch acanthoma in the pitch workers was $3.5 \%$ compared with $2.7 \%$ in the controls. However, on the two-year follow-up there was an increase in incidence in the pitch workers of $15.7 \%$. Only $10.4 \%$ of all pitch workers developed pitch acanthoma. The incidence was related not only to the duration of exposure but also to the degree of pitch contamination of the skin. The highest incidence was recorded in those with the greatest skin contamination, $24 \%$ compared with $3.2 \%$ in those with low exposure. Spontaneous regression was noticed in $26 \%$ of cases.

4. Photo-sensitivity: $57(4 \%)$ pitch workers recorded photo-sensitivity ('smarts'). There was no relationship between the incidence of 'smart' and pitch warts or acne.

5. Acneiform lesions: All acneiform lesions were much more common in the pitch workers $(93 \%)$ than in the controls $(31 \%)$.

6. Scrotal changes: $13.5 \%$ of all the pitch workers showed one or more of our recorded lesions. There was a $3.5 \%$ incidence of kerato-acanthoma and there was one squamous carcinoma.

In this series there is an increased susceptibility to some proliferative lesions, either premalignant or malignant, in pitch workers when compared with the controls. Only $10.7 \%$ of the working population were affected with pitch acanthoma and of these $50 \%$ had multiple warts. Even though there is an increasing incidence with increased exposure, this is not absolute and our findings suggest that there is also a personal susceptibility to pitch. 
Our interest was aroused in this subject by cases referred to us from a patent fuel works in the locality after routine six-monthly examinations by the works medical officer. At this works pitch and coal dust are fused by steam to make blocks or ovoids.

During the years 1957 to 1963 we saw 59 cases of hyperplastic squamous lesions. There were 35 pitch acanthomas (pitch warts), three squamous-cell carcinomas and 29 squamous keratoses or combinations of these lesions. The working population at risk was subject to slight variation, being approximately 150 persons. From these, six persons per year presented with pitch acanthoma, approximately $4 \%$ of the whole working population. It was well known by the men themselves that in many instances spontaneous resolution of pitch warts occurred, so that the total incidence of warts was probably higher. A history of multiple pitch warts was common, in fact $48 \%$ of the persons we saw with pitch warts had such a history of multiple warts and $23 \%$ had had five or more. There was definite knowledge of the date of onset of the first pitch acanthoma in nearly half of the men. In these, roughly half had had an exposure of up to 10 years before the first wart appeared, whilst the remainder had had exposures of between 10 and 20 years. One man was exposed for 50 years before the acanthoma appeared. These facts led us to carry out a control survey of all personnel in the fuel works to determine the true extent of pitch acanthoma and to relate this to pitch exposure in both time and degree. This was to be compared with a control population of the same age limit which had not been exposed to pitch.

\section{Investigation}

The two populations studied were comparable groups of men all aged between 20 and 69 years. Group A were pitch workers, totalling 144 persons; the whole working population was examined in 1963 and re-examined after a further two years. Group B was a control group totalling 263 persons in random selection from dermatological out-patients. It must be accepted, however, that such a control group might be expected to have a higher proportion of some diseases, for instance neoplasia, than a normal population. The age groups and racial distribution are shown in Table 1 and it will be seen that these are comparable. In addition we recorded the colouring of the hair and eyes. A full clinical examination of the skin was done for all proliferative and acneiform lesions and we recorded antecedent conditions referable to neoplasia, namely burns, scars, $x$-ray scars and virus warts. Biopsies were taken from suspicious proliferative lesions. In addition the ratio of exposure to pitch was estimated both in total time of exposure and in degree, the latter being graded as high (H), medium (M) and low (L) as judged by management and works medical authorities from the actual degree of contact with pitch at work. For example, pitch feeders (off-loading pitch) and press men (making cobbles) have high exposure. Maintenance men, such as electricians, who are at work
TABLE 1

Total Number of Persons Examined, PITCH WORKERS AND CONTROLS, AND RaCe AND Age Distribution

\begin{tabular}{|c|c|c|c|c|c|}
\hline & & & & Pitch workers & Controls \\
\hline \multicolumn{4}{|c|}{ Total no. examined } & 144 & 263 \\
\hline $\begin{array}{l}\text { Race } \\
\text { White } \\
\text { Indian } \\
\text { Negro... } \\
\text { Chinese }\end{array}$ & $\begin{array}{l}\cdots \\
\cdots \\
\cdots \\
\cdots\end{array}$ & $\begin{array}{l}\cdots \\
\cdots \\
\cdots\end{array}$ & $\begin{array}{l}\ldots \\
\ldots \\
\ldots \\
\cdots\end{array}$ & $\begin{array}{c}87.5 \% \\
5.5 \% \\
7.0 \% \\
-\end{array}$ & $\begin{array}{r}98.4 \% \\
0.8 \% \\
0.4 \% \\
0.4 \%\end{array}$ \\
\hline $\begin{array}{c}\text { Age group } \\
20-29 \quad \ldots \\
30-39 \quad \ldots \\
40-49 \quad \ldots \\
50-59 \quad \ldots \\
60-69 \quad \ldots\end{array}$ & $\begin{array}{l}\cdots \\
\cdots \\
\cdots \\
\cdots\end{array}$ & $\begin{array}{l}\cdots \\
\cdots \\
\cdots \\
\ldots\end{array}$ & $\begin{array}{l}\cdots \\
\cdots \\
\cdots \\
\ldots\end{array}$ & $\begin{array}{r}9.8 \% \\
22.2 \% \\
29.2 \% \\
27.0 \% \\
11.8 \%\end{array}$ & $\begin{array}{l}12.9 \% \\
18.6 \% \\
23.2 \% \\
24.8 \% \\
20.5 \%\end{array}$ \\
\hline
\end{tabular}

all over the factory have medium exposure, whereas crane drivers, boilermen and office staff have a low risk.

The clinical findings were classified into six main groups: (1) benign proliferative lesions (papillomata); (2) pre-malignant and malignant epidermoid lesions; (3) pitch acanthomas (pitch warts); (4) photo-sensitivity; (5) acneiform lesions; (6) scrotal changes; (7) antecedent and incidental or other skin lesions.

\section{Findings}

1. Benign proliferative lesions (papillomata)

The term 'papillomata' here refers to the local proliferation of the skin surface, basal or squamouscell in type. The term has been used in pitch workers to include small kerato-acanthoma which we regard as a distinct lesion. Papillomata were divided into four groups according to morphology (Hodgson, 1968): (a) flat shiny lesions; (b) sessile lesions, like small, smooth, plain warts in appearance; (c) pedunculated 'skin tags'; and $(d)$ plaques.

Flat, shiny and sessile lesions show a regular hyperplasia with hyperkeratosis. Their histological appearance is a regular acanthosis with hyperkeratosis.

Pedunculated 'skin tags' are fibro-epithelial polyps but some may be elongated tags of basal-cell papillomata.

Plaques are irregular basal-cell papillomata (seborrheic warts), often pigmented.

Incidence The overall incidence of all types in both populations increased with age. In the control group there were $4.1 \%$ in the age group $20-29$, reaching a maximum of $29 \%$ in the $50-59$ age group and decreasing to $25.7 \%$ in the $60-69$ years age group. In pitch workers there were $4.8 \%$ in the $20-29$ years 
age group, reaching a maximum of $36.2 \%$ in the $50-59$ years group, declining to $14 \%$ in the next decade. There were, however, more benign papillomata in the control group ( $73 \%$ ) than in the pitch workers $(58 \%)$. In both groups flat, shiny and sessile papillomata with regular hyperkeratotic hyperplasia occurred. These were mainly on the exposed skin of the hands and forearms in persons with blue eyes and fair hair. The highest incidence was between 50 and 59 years. Basal-cell papillomata of the plaque type (seborrheic warts) showed an increasing incidence with advancing age until $100 \%$ of those aged 80-100 years (in the control series) were affected.

2. Pre-malignant and malignant epidermoid lesions (excluding pitch acanthoma)

These we classified as follows:

Pre-malignant Squamous keratosis

Chronic tar dermatosis

Scars

$X$-ray scars

Malignant Squamous-cell carcinoma (epidermoid carcinoma)

Intra-epidermoid carcinoma (Bowen)

Basal-cell epithelioma (rodent ulcer).

There was an increased incidence of all squamous proliferative lesions in pitch workers. Particular findings were:

Squamous keratosis This was very slightly more common in the pitch workers, $12 \%$ compared with $10 \%$ in the control group. The areas affected were the exposed skin of the head and face, including the ears, forearms and hands. No palmar keratoses were noted.

Chronic tar dermatosis (Fisher, 1954) This was found in $5 \%$ ( 7 cases) of pitch workers. All seven of these had exposures which ranged from 30 to 50 years with an overall average of 43 years. In all there were eight persons in this series with over 40 years' exposure of whom three developed chronic tar dermatosis. There were 14 with 30 to 39 years' exposure and only two developed chronic tar dermatosis, the changes being skin thickening with hyperkeratosis, atrophy, scarring, pigmentary alterations (lentigines, poikilodermia, leuco-melanodermia), persisting erythema and telangectasia with hyperplastic proliferative and acneiform lesions. None of the controls showed these changes.

Scars Of the pitch workers $7 \cdot 7 \%$ showed obvious scars, mainly on the arms. These were possibly related to their occupation (wounds, pitch burns, radiation and treatment of acanthoma) and compared with an incidence of $2.2 \%$ in the controls, also probably traumatic in origin. No epidermoid carcinomata or kerato-acanthomas were seen to develop on scars.

X-ray Scars Five $(3.5 \%)$ of the pitch workers showed post-radiation scarring and two controls $(0.8 \%)$. No squamous neoplasia developed on these lesions.

Squamous-cell carcinoma (epidermoid carcinoma) These were more numerous in pitch workers $(2.8 \%)$ than in the controls $(0.4 \%)$. In the pitch workers, lesions developed on the scrotum in addition to those found on the exposed face and hands.

Intra-epidermoid carcinoma (Bowen's disease) No intra-epidermoid carcinomata were discovered in pitch workers; the controls showed an incidence of $0.4 \%$.

Basal-cell epithelioma (rodent ulcer) Pitch workers showed only occasional rodent ulcers, an incidence of $0.7 \%$ contrasting with a much higher rate of $12.5 \%$ in the control series. Over two consecutive year periods, 1966 and 1967, the incidence of rodent ulcers at the Cardiff Royal Infirmary dermatological out-patients was 49 in 7,858, an incidence of $0.69 \%$. Comparing the current incidence of basal-cell epithelioma and benign proliferative lesions in the control group we found that of the 33 cases, $91 \%$ of persons with rodent ulcers also had benign proliferative lesions and $48 \%$ had basal-cell papilloma (plaque type papilloma), whilst of the 230 without basal-cell epithelioma $71 \%$ showed benign proliferative lesions and $26 \%$ had basal-cell papilloma.

\section{Pitch acanthomas (pitch warts)}

Clinically and histologically all the pitch warts examined were true kerato-acanthomas. There was no clinical or histological difference in the acanthomas that developed in pitch workers from those in the controls who had not been exposed to pitch.

The majority were small in size, less than $2 \mathrm{~cm}$.

The tumours were common on the face, around the eyes and nose, on the ears and on the dorsum of the hands (Fig. 1). One was recorded on the foot and three on the scrotum. Two single giant tumours occurred, one on the forearm and one on the lower leg. These were not preceded by trauma.

At the initial examination the expected increase in incidence of acanthoma in pitch workers over the controls was marginal, $3.5 \%$ of pitch workers compared with $2.7 \%$ controls. However, on the two-year follow-up the incidence in pitch workers was much increased, $15.7 \%$ compared with $2.7 \%$ in the controls.

Only $10.4 \%$ of all the pitch workers developed pitch warts and these were all white-skinned 


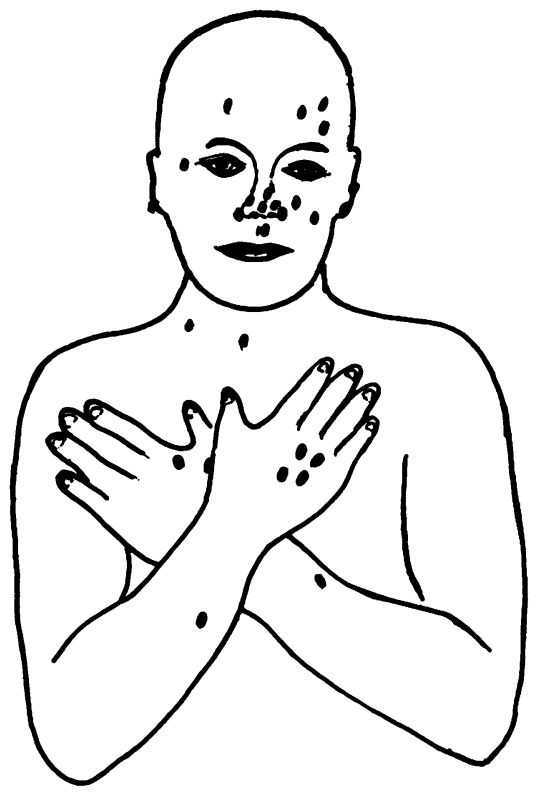

FIG. 1. Distribution of pitch acanthoma in 144 pitch workers.

persons although about $12.5 \%$ of the working population were Indians or negroes. There was, however, no relationship in the white workers to eye colour or hair as had been apparent in the benign papillomata. A spontaneous regression of pitch acanthomas was observed in $26 \%$.

The highest incidence of acanthomas was in persons aged 50-59 years among both the pitch workers and the controls. In pitch workers a higher percentage of 'warts' was seen in men over 60 who had had some 40 to 49 years' exposure. However, one man who had been exposed for 50 years had never had a 'wart' and another developed one after only three years (Fig. 2).

The incidence of acanthoma developing was influenced not only by the duration of exposure but also by the degree - high, medium or low - of the pitch hazard. The highest incidence was recorded in those workers with a combination of high, medium and low $(24 \%)$ and the least in those with low exposure $(3.2 \%)$ (Table 2 ).

Equation with other proliferative lesions showed that all such lesions were more common in pitch workers with acanthomas than in those without. Sessile papilloma was 3 times, squamous-cell carcinoma 5 times, squamous keratoses $4 \frac{1}{2}$ times and chronic tar dermatosis 10 times more common.

In attempting to assess the seasonal relationship of acanthoma by estimating the month of onset, it was found that $70 \%$ (21 acanthomas) occurred

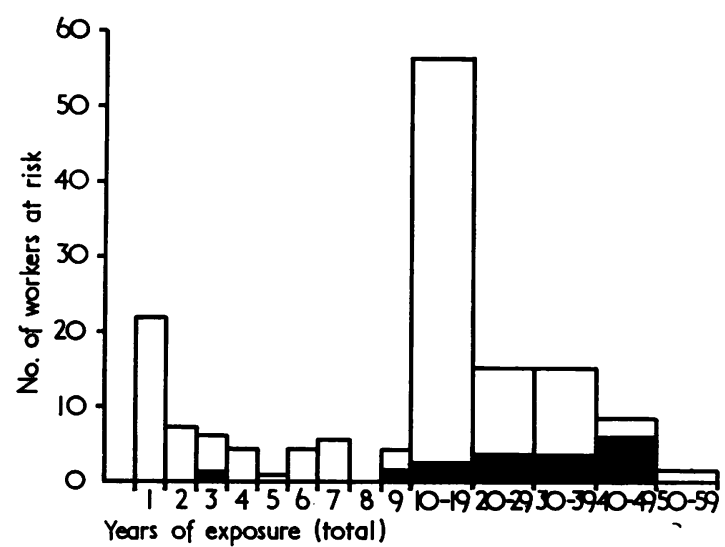

FIG. 2. Distribution of total exposure in 144 pitch workers and number of workers who had pitch warts (shaded area).

between April and September, the lighter months of the year.

\section{Photo-sensitivity}

Photo-sensitivity from pitch with acute erythema, known to the men as the 'smarts', was estimated from the history. One man was seen with an acute photo contact vesicular bullous dermatitis during the summer. Most showed sunburn erythema. It was not possible accurately to classify 'the smarts' into degrees or to estimate the duration of its continuance when away from contact. Some men, however, stated that it continued to persist for some weeks on holiday in the summer. Cold winds often caused increased smarting discomfort; $57.5 \%$ of pitch workers (83 persons) recorded 'smarts' whilst $42.5 \%$ (61 persons) had no 'smarts'.

There were no 'smarts' recorded in Indian or negro workers. In white-skinned persons, 'smarts' arose most commonly in those with relatively lightly pigmented skin.

There was no relationship between the incidence of 'smarts' and kerato-acanthoma or acne.

TABLE 2

Number of Persons at Risk, Type of EXPOSURE AND NUMBER OF WORKERS WHO DEVELOPED ACANTHOMA

\begin{tabular}{cc|c|c|c}
\hline $\begin{array}{c}\text { Type of } \\
\text { exposure }\end{array}$ & No. at risk & $\begin{array}{c}\text { No. of } \\
\text { acanthomas }\end{array}$ & \% Incidence \\
\hline H + M + L & $\ldots$ & 33 & 8 & 24 \\
M + L. &. & 48 & 6 & $12 \cdot 5$ \\
L.. &. & 63 & 2 & $3 \cdot 2$ \\
\hline
\end{tabular}




\section{Acneiform lesions}

Four groups of acneiform lesions were recorded: (a) comedones; (b) acne; (c) sebaceous retention cysts; $(d)$ folliculitis.

Such lesions were present alone, in combination or all together as fully developed acnes of constitutional or occupational aetiology. Folliculitis included lesions also on the body and limbs.

Incidence All acneiform lesions were much commoner in pitch workers $(93 \%)$ than in controls $(31 \%)$ (Fig. 3). For example, comedones were four times more common and acne twice as common. The greatest differences were found with retention cysts and folliculitis, both of which were nine times more common in pitch workers.

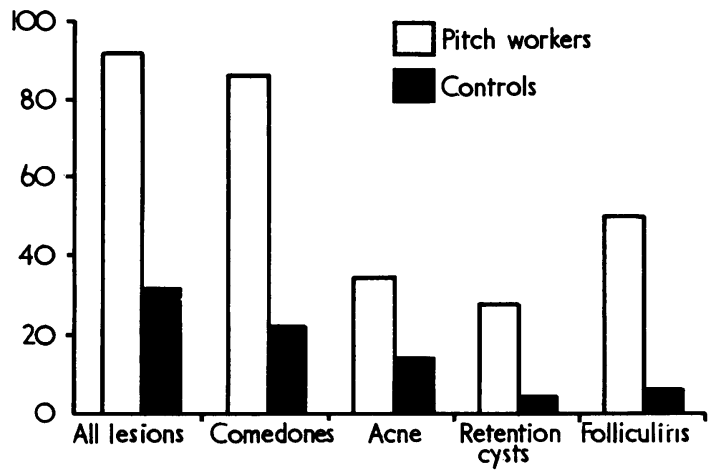

FIG. 3. Incidence of all acneiform lesions and incidence in separate groups for pitch workers and controls.

The incidence of all lesions according to age groups in the pitch workers rose from $9.7 \%$ in the $20-29$ years group to a peak of $27.8 \%$ in the $40-49$ years group and then declined. In the control group the incidence remained constant at between $6.5 \%$ and $6.9 \%$ during this period and then declined. However, in neither population was there any direct relationship between the development of keratoacanthoma and acneiform lesions. Nor was there any relation with folliculitis alone, from simple soiling of the skin and poor personal hygiene. Out of the total of 27 kerato-acanthomas only five developed in pitch workers with folliculitis. Also there was no apparent association between keratoacanthoma and any photo-sensitivity which might relate to increased soiling and absorption of pitch dust into sebum of acne skin.

'Smarts' occurred with nearly equal frequency in those with acne ( 32 persons $-64 \%)$ and in those without (94 persons $-54 \%$ ).

\section{Scrotal changes}

Because of the particular interest in scrotal changes our observations are recorded separately. We record that all the $4.2 \%$ ( 8 persons) of pitch workers who showed texture changes (velvety plaques of apparently thickened skin) also had kerato-acanthomas elsewhere and five of these persons also had chronic tar dermatosis on other areas of the skin. In addition, any special pigment anomalies and unusual texture changes were noted. These texture changes are recorded as velvety patches of apparently thickened skin. No biopsies were performed. The scrotum was not examined in the controls.

In the pitch workers the lesions were as follows:

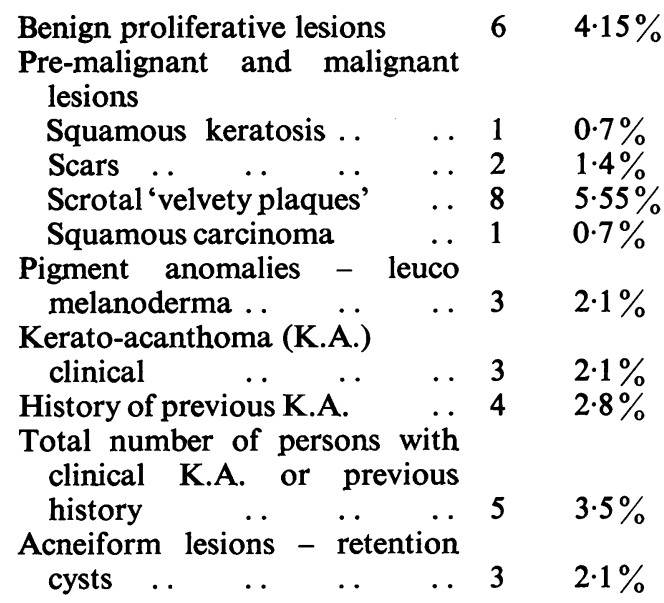

Relation of kerato-acanthoma, pigment anomalies and velvety plaques to proliferative lesions elsewhere on body The one man with squamous keratosis showed poikilodermia. The kerato-acanthomas were confirmed histologically. All 5 persons had had kerato-acanthomas elsewhere. Two persons showed poikilodermia elsewhere. All 3 persons with pigment alterations showed poikilodermia or chronic tar dermatosis elsewhere.

Of the 8 persons who had scrotal velvety plaques, all 8 showed kerato-acanthoma elsewhere, 3 showed kerato-acanthoma also on the scrotum; 5 showed chronic tar dermatosis elsewhere; and 3 showed the velvety scrotal plaques alone. All these persons had had a high exposure to pitch and a total exposure duration of from 12 to 50 years.

Comment Of the pitch workers, $13.5 \%$ showed scrotal proliferative changes. Kerato-acanthoma occurred on the scrotum, and all those affected also showed kerato-acanthoma elsewhere. Pigment alterations which affect the skin elsewhere in pitch workers also affect the scrotum.

Scrotal velvety plaques, which may be the result of frictional and/or chemical inflammation or hyper- 
plasia, have a definite association with proliferative lesions caused by pitch elsewhere, in fact all persons with this change in the scrotal skin had had keratoacanthoma elsewhere and five showed evidence of chronic tar dermatosis elsewhere.

\section{Antecedent and incidental skin lesions}

During the examination other conditions were recorded which might possibly be relevant.

Virus warts were more frequent in pitch workers $(11.1 \%, 16)$ than in the controls $(4.2 \%, 11)$. In the pitch workers infected with virus warts only one pitch acanthoma $(6.25 \%)$ was found, whilst in 128 men without virus warts 4 acanthomas $(3.1 \%)$ were found. Photosensitivity did not appear to influence the development of virus warts.

Other lesions recorded are shown in Table 3.

TABLE 3

Developed Acanthoma

\begin{tabular}{|c|c|c|c|c|c|}
\hline \multicolumn{4}{|c|}{ Dermatosis } & $\begin{array}{c}\text { Pitch } \\
\text { workers }\end{array}$ & Controls \\
\hline $\begin{array}{l}\text { Eczema } \\
\text { Psoriasis } \\
\text { Rosacea } \\
\text { Acne keloid }\end{array}$ & $\begin{array}{l}\cdots \\
\cdots \\
\cdots \\
.\end{array}$ & $\begin{array}{l}\cdots \\
\cdots \\
\cdots \\
\cdots\end{array}$ & $\begin{array}{l}\ldots \\
\ldots \\
\ldots \\
\ldots\end{array}$ & $\begin{array}{cc}15 \cdot 2 \% & (12) \\
\text { Nil } & \\
1.4 \% & (2) \\
2 \cdot 1 \% & (3)\end{array}$ & $\begin{array}{r}20.9 \%(55) \\
4.9 \%(13) \\
1.5 \%(4) \\
1.3 \% \quad(3)\end{array}$ \\
\hline Total no. of & 150 & $n g$ & & 144 & 263 \\
\hline
\end{tabular}

\section{Discussion}

\section{Proliferative lesions of all types}

It seems that pitch exposure does not influence the incidence or the site of development of benign proliferative lesions but it is interesting to note that in spite of the increasing incidence in age of all types, the sessile papillomata on the hands and forearms reach a peak incidence between 50 and 60 years of age both in the pitch workers and in non-pitchexposed persons.

Pre-malignant conditions, especially chronic tar dermatosis, were much more common in the pitch workers. Actinic squamous keratosis showed little difference in incidence in spite of the photosensitivity induced by pitch. The higher incidence of scars reflects traumata at work but this did not seem to influence the development of neoplasia: $4.2 \%$ of pitch workers showed some texture changes in the scrotal skin. In the absence of histological confirmation of proliferative changes in the velvety thickened areas, it is possible that these might be inflammatory due either to friction of pitchcontaminated clothing or to an irritant chemical effect of pitch. We feel that they should be regarded with a high degree of suspicion and that this type of change should be kept under continued observation.
Carcinoma of the scrotum has been accepted as primarily occupational since the days of Percival Pott (1775). We confirm that kerato-acanthoma can occur on the scrotum as it does on other hair-bearing sites in addition to squamous carcinoma. In our series the accepted risk of epidermoid carcinoma on other sites is confirmed by the incidence of $2.8 \%$ in pitch workers compared to $0.4 \%$ in the controls. The very high incidence of basal-cell carcinoma in the controls must reflect the selection of patients, those with basal-cell carcinoma being referred specially. Lever (1961) considers that this tumour occurs on light-exposed skin. In this series in the summer months there is no suggestion that photosensitivity from pitch, as judged by 'smarts', is a factor. We confirm that kerato-acanthomas (pitch warts) follow the pattern of distribution on the areas of short hairs and the increased incidence of multiple tumours in pitch workers, and that these regressed spontaneously in $26 \%$ of persons. These facts are well known.

It is a fact that only $10.4 \%$ of persons at risk developed the pitch warts.

A genetic or family susceptibility appears to exist in multiple non-occupational kerato-acanthoma of the self-healing Ferguson Smith (1948) type. The fact that ours were multiple does not reflect entirely a genetic influence because of their occurrence on pitch-exposed areas and the increasing incidence with increasing degree of exposure. The Ferguson Smith type also occurs in early life but in this series the peak incidence was in the 50-59 years age group.

All the pitch warts were seen on white-skinned persons although $12.5 \%$ of the working population were coloured or black-skinned. This accords with the findings of others, although kerato-acanthoma is known to develop in such skins.

\section{Acneiform lesions}

The preponderance of acneiform lesions, comedones, acne, sebaceous retention cysts and folliculitis in the pitch workers $(93 \%$ compared to $31 \%$ in the controls) seems to indicate a general soiling action on the skin rather than a relation to any proliferative action of the pitch. The four-fold increased incidence of comedones and the nine-fold increased incidence of retention cysts and folliculitis can be explained on the grounds of either a soiling obstruction or an irritant effect on the pilosebaceous follicle orifice.

This is supported by the continued higher incidence in pitch workers in late life $(27.8 \%)$ in the 40-49 years age group compared with $6.9 \%$ in the controls at a time when male acne of constitutional origin would normally be declining.

\section{Conclusions}

It is probable that in most neoplasia the development 
of the tumour is influenced by the carcinogenic agent and by personal susceptibility. The role of pitch in inducing epidermal neoplasia is without dispute. Other accepted initiators of carcinogenesis in man are hydrocarbons derived from petroleum oil, trauma and radiant energy, particularly ultraviolet light and radio-activity. None of our workers gave a history of any substantial industrial exposure to mineral oils and no lesions were recorded at the site of previous trauma or scars or on the site of previous radiation damage.

Although ultra-violet light is a known promoter of epidermoid carcinoma from environmental exposure and three such carcinoma tumours occurred in this series of pitch workers, one of these occurred on non-light exposed skin on the scrotum. Keratoacanthomas occurred mostly on the exposed skin, and all were in white-skinned persons and apparently mostly in the lighter months of April to September. On the other hand, there was no apparent relationship to photo-sensitivity induced by pitch, and no increased incidence in blond or red-headed persons with a poor light tolerance. Kerato-acanthomas were also found occasionally on unexposed skin. There was also no significant difference in the incidence of photo-sensitivity in those with acne and in those without. One cannot altogether exclude the effect of ultra-violet light in the causation of epidermal carcinoma and pitch acanthoma in this series, but we regard pitch as the main initiator.

A virus has been postulated as initiating keratoacanthoma (Zelickson, 1962). We have not done any viral studies and can only record that clinically the incidence of acanthotic tumours due to the virus wart appeared to be more common in the pitch workers than in the controls.

\section{Personal factors}

Among the important personal factors that appear to determine the development of pitch acanthoma are race, anatomical distribution and constitutional factors suggesting susceptibility. Although acanthoma can develop in coloured skins, all our pitch acanthomas affected white skin Caucasian persons in spite of the fact that over $10 \%$ of the working population were coloured. The anatomical distribution fits in well with the previously described distribution on skin areas of the short soft hairs. It has been suggested that the keratoacanthoma is initiated in the outer root sheath of the hair follicle and the full period of growth to spontaneous remission ( $26 \%$ in our series) is analogous to the rhythm of the hair cycle.

In this series a susceptibility to all proliferative lesions, both benign and malignant, was observed in pitch workers. This is reflected here in the apparent susceptibility to pitch acanthoma. In this series only $10.7 \%$ of the working population were affected and $50 \%$ of those had multiple warts. Although there was a gradually increasing incidence with duration and degree of exposure, this is not absolute and some persons developed these rapidly, others after a short exposure, whereas others did not develop them at all. In our view, however, these facts strongly suggest a personal susceptibility to pitch acanthoma as well as to the other proliferative lesions. Our $10.7 \%$ of 'susceptibles' appear to be of the same order as the 1 in 8 heavy cigarette smokers who die of lung cancer (Leading article, 1957). In view of this risk it would seem reasonable to afford the working population maximum protection against this hazard.

\section{Recommendations for control of hazard}

Although many facilities already exist, a considered programme might include these points, if they are not already in operation:

\section{(a) Reduction of pitch exposure}

Pitch should be delivered in a molten state by tanker, if this is practicable, instead of in a dry, hardened, solid state likely to increase a dust hazard on being broken.

\section{(b) Susceptible persons}

Susceptible persons (those prone to develop pitch 'warts' or other proliferative lesions, benign or malignant) should be removed from excessive exposure and selective employment of estimated more resistant persons should be encouraged.

\section{(c) Personal hygiene}

Clean industrial protective clothing and adequate ablution facilities and time should be provided. Further research is required into methods of proper cleansing of pitch soiling of the skin having regard to solubility in sebum and its cutaneous staining properties.

The authors thank the National Coal Board for the facilities made available.

\section{References}

Fisher, R. E. W. (1954). Skin cancer in tar workers. Trans. Ass. industr. med. Offrs, 3, 315-318.

Hodgson, G. A. (1968). Some degenerative and proliferative skin disorders of the aged. Geront. clin. (Basel), 10, 201-214.

Leading article (1957). Dangers of cigarette-smoking. Brit. med. J., $1,1518$.

Lever, W. F. (1961). Histopathology of the Skin, 3rd. ed., p. 476. Lippincott, Philadelphia.

Pott, P. (1775). Chirurgical Works, Vol. 5, p. 93. London.

Smith, J. Ferguson (1948). Multiple primary, self-healing squamous epithelioma of the skin. Brit.J. Derm., 60, 315-318.

Zelickson, A. S. (1962). Virus-like particles demonstrated in keratoacanthomas by electron microscopy. Acta derm.-venereol (Stockh.), 42, 23-26.

Received for publication July 23, 1969. 